\title{
Spin-polarization and electronic properties of half-metallic Heusler alloys calculated from first-principles
}

\author{
I Galanakis† and Ph Mavropoulos \\ † Department of Materials Science, School of Natural Sciences, University of \\ Patras, Patras 265 04, Greece \\ ‡Institut für Festkörperforschung, Forschungszentrum Jülich, D-52425 Jülich, \\ Germany \\ E-mail: galanakis@upatras.gr,ph.mavropoulos@fz-juelich.de
}

\begin{abstract}
Half-metallic Heusler alloys are amongst the most promising materials for future magnetoelectronic applications. We review some recent results on the electronic properties of these compounds. The origin of the gap in these half-metallic alloys and its connection to the magnetic properties are well understood. Changing the lattice parameter shifts slightly the Fermi level. Spinorbit coupling induces states within the gap but the alloys keep a very high degree of spin-polarization at the Fermi level. Small degrees of doping and disorder as well as defects with low formation energy have little effect on the properties of the gap, while temperature effects can lead to a quick loss of half-metallicity. Finally we discuss two special issues; the case of quaternary Heusler alloys and the half-metallic ferrimagnets.
\end{abstract}

PACS numbers: $75.47 . \mathrm{Np}, 71.20 . \mathrm{Be}, 71.20 . \mathrm{Lp}$

Submitted to: J. Phys.: Condens. Matter 


\section{Half-metallic Heusler Alloys}

The recent development in electronics, combining the magnetic and semiconducting materials (so-called magnetoelectronis or spintronics), has brought half-metallic ferromagnets, initially predicted by de Groot and collaborators in 1983 [1], to the centre of scientific research. These magnetic metals have the peculiarity that the minority-spin band-structure is semiconducting while the majority-spin bandstructure is metallic. Such half-metals exhibit, ideally, a 100\% spin polarization at the Fermi level and therefore these compounds should have a fully spin-polarized current and should be ideal spin injectors into a semiconductor, thus maximizing the efficiency of spintronic devices [2].

An important part of the scientific effort has been focused on the study and fabrication of half-metallic Heusler alloys. The first family of Heusler alloys studied were of the form $\mathrm{X}_{2} \mathrm{YZ}$, crystallizing in the $L 2_{1}$ structure which consists of four fcc sublattices, where $\mathrm{X}$ a high valent transition or noble metal atom, $\mathrm{Y}$ a lowvalent transition metal atom and $\mathrm{Z}$ an sp element [3, 4. Such Heusler compounds have attracted a lot of interest due to the possibility to study in the same family of alloys a series of interesting diverse magnetic phenomena like itinerant and localized magnetism, antiferromagnetism, helimagnetism, Pauli paramagnetism or heavy-fermionic behaviour [3]. The Heusler alloys of the second class are of the form $\mathrm{XYZ}$, crystallizing in the $C 1_{b}$ structure, and consisting of three fcc sublattices; they are often called half- or semi-Heusler alloys in literature, while the $L 2_{1}$ compounds are referred to as full Heusler alloys. The interest in these types of intermetallic alloys was revived after the prediction [1, using first-principles calculations, of half-metallicity in NiMnSb, a half-Heusler compound.

The main advantages of Heusler alloys with respect to other half-metallic systems (e.g. some oxides like $\mathrm{CrO}_{2}$ and $\mathrm{Fe}_{3} \mathrm{O}_{4}$ and some manganites like $\mathrm{La}_{0.7} \mathrm{Sr}_{0.3} \mathrm{MnO}_{3}$ ) [5]) are their relatively high Curie temperatures 3, 4. While for the other compounds the Curie temperature is near the room temperature, e.g. for NiMnSb it is $730 \mathrm{~K}$ and for $\mathrm{Co}_{2} \mathrm{MnSi}$ it reaches the $985 \mathrm{~K}[\underline{3}$. and their structural similarity to the zincblende structure, adopted by binary semiconductors widely used in industry (such as GaAs on $\mathrm{ZnS}$ ). Heusler alloys have been already incorporated in spin-filters [6], tunnel junctions [7, and GMR devices [8]. The most successful recent applications in spintronics concern the half-metallic full Heusler alloys. The group of Westerholt has incorporated $\mathrm{Co}_{2} \mathrm{MnGe}$ in the case of spin-valves and multilayer structures 9]. The group of Reiss managed to create magnetic tunnel junctions based on $\mathrm{Co}_{2} \mathrm{MnSi} 10$.11. A similar study by Sakuraba and collaborators resulted in the fabrication of magnetic tunnel junctions using $\mathrm{Co}_{2} \mathrm{MnSi}$ as magnetic electrodes and $\mathrm{Al}-\mathrm{O}$ as the barrier and their results are consistent with the presence of half-metallicity for $\mathrm{Co}_{2} \mathrm{MnSi}$ [12] Dong and collaborators recently managed to inject spin-polarized current from $\mathrm{Co}_{2} \mathrm{MnGe}$ into a semiconducting structure [13].

In a recent article 14 we have reviewed theoretical results of the basic electronic and magnetic properties of the half-metallic Heusler alloys. The gap has its origin for both the half- and full-Heusler alloys in the $d$ - $d$ hybridisation, which is fundamental for understanding their electronic and magnetic properties. For both families of compounds the total spin magnetic moment scales with the number of valence electrons and can be described by a Slater-Pauling rule.

In the present contribution we review our most recent results on the electronic properties of these alloys obtained from first-principles electronic structure 
calculations. In section 2 we summarize our older results (see references [14 and [15] for an extended review). The following sections are dedicated to phenomena which can destroy half-metallicity. In particular section 3 discuss the effect of the lattice parameter, section 4 is dedicated to the effect of spin-orbit coupling, section 5 to the doping and disorder effects and the formation of defects. In sections 6 and 7 we discuss two special cases: the quaternary Heusler compounds and the case of halfmetallic ferrimagnets, respectively. In section 8 we review the theoretical studies on the behaviour of the half-metallicity and of the magnetization as a function of the temperature. Finally in section 9 we summarize and conclude our review. Note that the problems of surface/interface states [16, 17] are reviewed in other papers in this volume.

To obtain these results we have employed a variety of electronic structure methods each one suitable for the property under investigation. The results in section 2 3 and 廿 4 have been obtained using the full-potential version of the screened Korringa-KohnRostoker (KKR) method [18]. The relativistic calculations to produce the orbital moments in 4 are performed with the fully-relativistic version of the KKR method where the Dirac equation is solved [19]. Results in sections 5 and 7 come from the full-potential nonorthogonal local-orbital minimum-basis band structure scheme (FPLO) [20]. Finally for the study of the quaternary Heusler alloys the KKR method is employed within the coherent potential approximation (CPA) to simulate the disorder in the crystal 21].

\section{Electronic and gap properties - Slater Pauling behaviour}

The electronic, magnetic and gap properties of half-Heusler alloys have been extensively studied in reference [22] and of full-Heusler compounds in reference 23. These results have been extensively also reviewed in reference 14 and in the introductory chapter of [15] and the reader is directed to them for an extended discussion. In this section we will only briefly overview these properties.

Several Heusler alloys have been predicted to be half-metals 24]; perhaps the most widely studied among them is NiMnSb, the first to be predicted as a half-metal. The electronic band-structure calculations of NiMnSb reveal that for both spin directions there is one band at around $-12 \mathrm{eV}$ with reference to the Fermi level which arises from the $s$ states of the $\mathrm{Sb}$ atom. The next three lowest bands are due to the $p$ states of $\mathrm{Sb}$ and accommodate also electrons from the transition metal atoms 22. The $\mathrm{Ni}$ and Mn majority-spin $d$ states are energetically close and form a common band. The situation is different for the minority-spin states: due to the strong exchange splitting of $\mathrm{Mn}$, the minority Mn $d$-states are unoccupied, while the ones of $\mathrm{Ni}$ are occupied. The minority-spin gap arises between the bonding and antibonding $d$-hybrids created among the Mn and Ni states (in this respect, the origin of the gap is similar to the one in compound semiconductors like GaAs or ZnSe). The minority-spin bonding states have most of their weight at the $\mathrm{Ni}$ atom and are occupied, while the antibonding states have their weight mostly at the $\mathrm{Mn}$ atom and are unoccupied. This leads to very strong localized spin moments at the Mn atoms (see table $\square$ for the values of the moments) 25. A simple band counting shows that the total spin moment is exactly 4 $\mu_{\mathrm{B}}$ for NiMnSb; this is a secial case of the Slater-Pauling behaviour, leading to integer moments for half-metallic systems.

The total moment of the half-metallic $C 1_{b}$ Heusler alloys follows a simple rule. The total number of electrons, $Z_{t}$, is given by the sum of the number of spin-up 

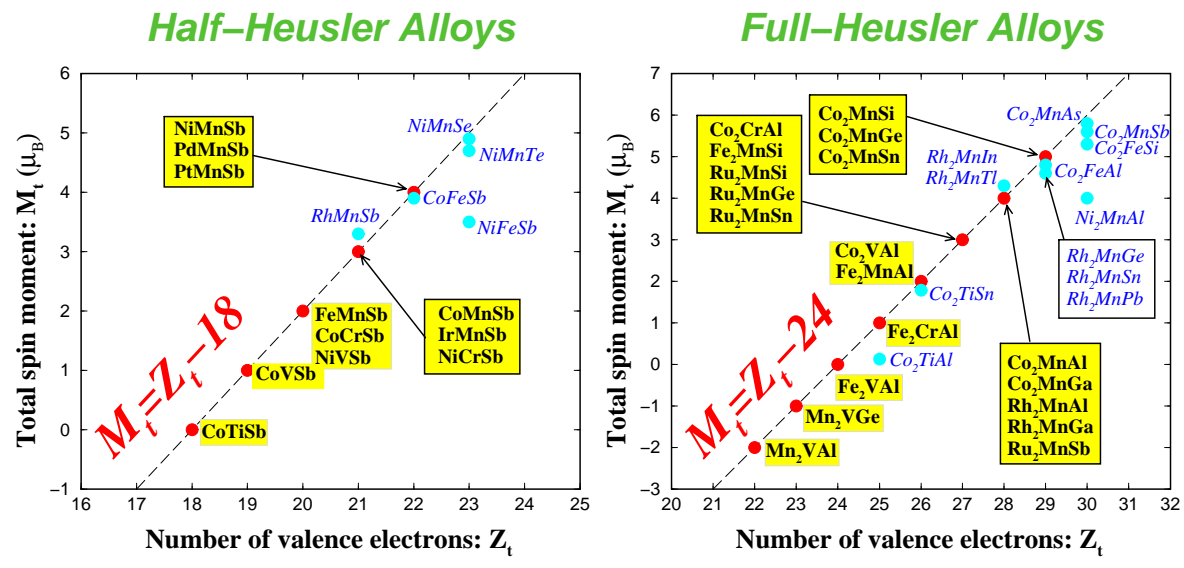

Figure 1. (Color online) Calculated total spin moment per unit cell as a function of the total number $Z_{t}$ of valence electrons per unit cell for all the studied half (left panel) and full (right panel) Heusler alloys. The dashed line represents the Slater-Pauling behaviour.

and spin-down electrons, while the total moment $M_{t}$ per unit cell is given by the difference. Since 9 minority bands are fully occupied, we obtain the simple "rule of 18 " for half-metallicity in $C 1_{b}$ Heusler alloys 22, 27.

$$
M_{t}=Z_{t}-18 \text {. }
$$

This behaviour of the total moment resembles the Slater-Pauling behaviour of the binary transition metal alloys with the difference that in the latter ones the total spin moment decreases with the number of valence electrons 28. The half-Heusler alloys with 18 valence electrons (e.g., CoTiSb) are nonmagnetic semiconductors, where the gap is again created by the $d-d$ hybridization.

In figure 1(left panel) we have gathered the calculated spin magnetic moments per formula unit for the half-Heusler alloys which we have plotted as a function of the total number of valence electrons. The dashed line represents the rule $M_{t}=Z_{t}-18$ obeyed by these compounds. The total moment $M_{t}$ is an integer (in $\mu_{B}$ ), assuming the values $0,1,2,3,4$ and 5 if $Z_{t} \geq 18$. The value $M_{t}=0$ corresponds to the semiconducting phase and the value $M_{t}=5 \mu_{B}$ to the maximal moment when all 5 majority $d$-states are filled. Firstly we varied the valence of the lower-valent (i.e. magnetic) transition metal atom. Thus we substitute $\mathrm{V}, \mathrm{Cr}$ and $\mathrm{Fe}$ for $\mathrm{Mn}$ in the $\mathrm{NiMnSb}$ and CoMnSb compounds using the experimental lattice constants. For all these compounds we find that the total spin moment scales accurately with the total charge and that they all present half-metallicity.

In the second part of this section we discuss the properties of the full-Heusler alloys like $\mathrm{Co}_{2} \mathrm{MnSi}$ and $\mathrm{Co}_{2} \mathrm{CrAl}$. Their electronic and magnetic properties are similar to the half-Heusler compounds with the additional complication of the presence of $2 \mathrm{Co}$ atoms per unit cell. In addition to the bands the half-Heusler alloys, there are five states exclusively located at the Co sites and near the Fermi level [23]. Now, the Fermi level is located among these states so that three out of five are occupied and two of them unoccupied leading to smaller gaps. Now there are 12 minority-spin occupied states and the compounds with 24 valence electrons like $\mathrm{Fe}_{2} \mathrm{VSb}$ are semiconductors. If $\mathrm{V}$ is substituted by a higher-valent atom, spontaneous spin polarization occurs, and 
the exchange splitting shifts the majority states to lower energies. Thus the extra electrons fill in only majority states: $\mathrm{Co}_{2} \mathrm{CrAl}$ (27 valence electrons) has a total spin moment of $3 \mu_{\mathrm{B}}$ and $\mathrm{Co}_{2} \mathrm{MnSi}$ (29 valence electrons) a spin moment of $5 \mu_{\mathrm{B}}$ as can be seen in table

The presence of the second Co atom has also another important consequence. Now each Mn atom has an octehedral environment of Co atoms as first neighbours and not four as was the case for CoMnSb. This leads to an increased hybridization between the $d$ orbitals of the $\mathrm{Mn}$ and $\mathrm{Co}$ atoms and the spin moment of $\mathrm{Co}$ in $\mathrm{Co}_{2} \mathrm{MnSi}$ is slightly lower than $1 \mu_{\mathrm{B}}$, considerably larger than the Co moment in CoMnSb.

Following the above discussion we investigate the Slater-Pauling behaviour with the "rule of 24 " now instead of 18 in the half-Heuslers :

$$
M_{t}=Z_{t}-24 .
$$

In figure 1 (right panel) we have plotted the total spin moments for all the studied compounds as a function of the total number of valence electrons. The dashed line represents the Slater-Pauling rule of half-metallic full Heusler alloys. The "magical number 24" arises from the fact that the minority band contains 12 electrons per unit cell: 4 are occupying the low lying $s$ and $p$ bands of the $s p$ element and 8 the Co-like minority $d$ bands (the Mn-Co bonding hybrids: $2 \times e_{g}$ and $3 \times t_{2 g}$, and the non-bonding Co states localized only at the Co sites: $\left.3 \times t_{1 u}\right)$. Since 7 minority bands $\left(2 \times \operatorname{Co} e_{u}\right.$, $5 \times \mathrm{Mn} d)$ are unoccupied, the largest possible moment is $7 \mu_{B}$ and would occur if all majority $d$-states were occupied. Of course it would be impossible to get a compound with a total spin moment of $7 \mu_{B}$ but even $M_{t}=6 \mu_{B}$ is difficult to obtain. As it was shown by Wurmehl et al. 29], the on-site correlations in $\mathrm{Co}_{2} \mathrm{FeSi}$ play a critical role for this compound and calculations within the LDA $+\mathrm{U}$ scheme, rather than the LDA, give a spin moment of $6 \mu_{B}$.

The exchange interactions, stabilizing ferromagnetism, have been studied in reference [30] using the adiabatic (frozen-magnon) approximation to calculate the inter-atomic exchange parameters for the half-Heusler alloys $\mathrm{NiMnSb}$ and $\mathrm{CoMnSb}$. The dominant interaction is between the $\mathrm{Mn}$ atoms. The magnetic interactions are more complex in full-Heusler alloys $\mathrm{Co}_{2} \mathrm{MnSi}$ and $\mathrm{Co}_{2} \mathrm{CrAl}$ [30, 31]. In both cases, ferromagnetism is stabilized by the inter-sublattice interactions between the $\mathrm{Mn}(\mathrm{Cr})$ and Co atoms and between Co atoms belonging to different sublattices.

Before closing this section we discuss also the role of the $s p$-elements in halfmetallic heusler alloys. While the $s p$-elements are not responsible for the appearance of the minority gap, they are nevertheless very important for the physical properties of the Heusler alloys and the structural stability of the $C 1_{b}$ structure. There are three important features:

(i) While an $\mathrm{Sb}$ atom has 5 valence electrons $\left(5 s^{2}, 5 p^{3}\right)$, in the NiMnSb compound each $\mathrm{Sb}$ atom introduces a deep lying $s$-band, at about $-12 \mathrm{eV}$, and three $p$-bands below the centre of the $d$-bands. These bands accommodate a total of 8 electrons per unit cell, so that formally $\mathrm{Sb}$ acts as a triply charged $\mathrm{Sb}^{3-}$ ion. Analogously, a Te-atom behaves in these compounds as a $\mathrm{Te}^{2-}$ ion and a Sn-atom as a $\mathrm{Sn}^{4-}$ ion. This does not mean, that locally such a large charge transfer exists. In fact, the $s$ - and $p$-states strongly hybridize with the transition metal (TM) $d$-states and the charge in these bands is delocalized and locally $\mathrm{Sb}$ even loses about one electron, if one counts the charge in the Wigner-Seitz cells. What matters here is that the $s$ - and $p$-bands accommodate 8 electrons per unit cell, thus reducing the electrons which have to be accommodated by the $d$-bands of the TM atoms. 

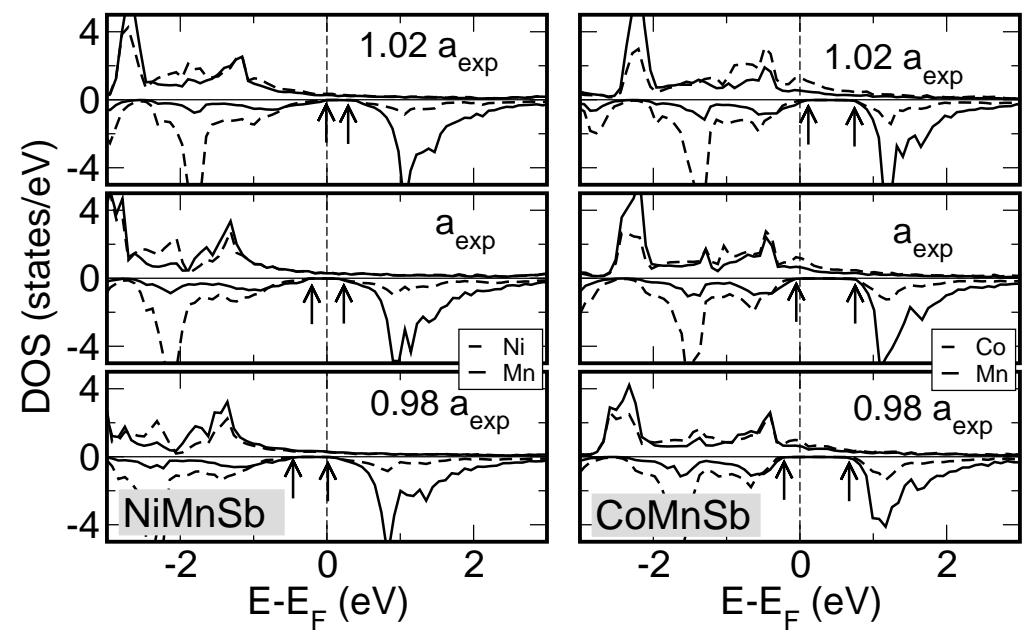

Figure 2. Atom-resolved density of states (DOS) for the experimental lattice parameter for $\mathrm{NiMnSb}$ and $\mathrm{CoMnSb}$, compared with the once compressed or expanded by $2 \%$. Arrows indicate the edges of the minority gap.

(ii) The $s p$-atom is very important for the structural stability of the Heusler alloys. For instance, it is difficult to imagine that the calculated half-metallic NiMn and PtMn alloys with zinc-blende structure actually exist, since metallic alloys prefer highly coordinated structures like fcc, bcc, hcp etc. Therefore the $s p$-elements are decisive for the stability of the $C 1_{b}$ compounds. A careful discussion of the bonding in these compounds has been recently published by Nanda and Dasgupta [26] using the crystal orbital Hamiltonian population (COHP) method. For the semiconductor $\mathrm{FeVSb}$ they find that, while the largest contribution to the bonding arises from the V- $d$ - Fe- $d$ hybridization, contributions of similar size arise also from the Fe- $d$ - Sb- $p$ and the V- $d-\mathrm{Sb}-p$ hybridization. Similar results are also valid for the semiconductors CoTiSb and NiTiSn and in particular for the half-metal NiMnSb. Since the majority $d$-band is completely filled, the major part of the bonding arises from the minority band, so that similar arguments as for the semiconductors apply.

(iii) Another property of the $s p$-elements is worthwhile mentioning: substituting the $\mathrm{Sb}$ atom in NiMnSb by $\mathrm{Sn}, \mathrm{In}$ or Te destroys the half-metallicity [22. This is in contrast to the substitution of $\mathrm{Ni}$ by $\mathrm{Co}$ or $\mathrm{Fe}$, which is documented in table 1 The total moment of $4 \mu_{B}$ for NiMnSb is reduced to $3 \mu_{B}$ in CoMnSb and $2 \mu_{B}$ in FeMnSb, thus preserving half-metallicity. In NiMnSn the total moment is reduced only to $3.3 \mu_{B}$ (instead of 3 ) and in NiMnTe the total moment increases only to $4.7 \mu_{B}$ (instead of 5). Thus by changing only the $s p$-element it is rather difficult to preserve the half-metallicity, since the density of states changes more like in a rigid band model 22 .

\section{Effect of the lattice parameter}

Changes of the lattice parameter can influence the electronic and magnetic properties of the $C 1_{b}$ and $L 2_{1}$ Heusler alloys. To the best of our knowledge no relevant experimental study exists. In figure 2 we show the $\mathrm{DOS}$ of $\mathrm{NiMnSb}$ and $\mathrm{CoMnSb}$ for the experimental lattice parameter and the ones compressed and expanded by 2 
Table 1. Calculated spin $\left(m_{\text {spin }}\right)$ and orbital $\left(m_{\text {orb }}\right)$ magnetic moments in $\mu_{B}$ for the XMnSb half-Heusler and $\mathrm{X}_{2} \mathrm{YZ}$ full-Heusler compounds. The last three columns are the total spin and orbital magnetic moment and their sum, respectively. The indexes $\mathrm{X}, \mathrm{Y}, \mathrm{Z}$ refer to the corresponding atoms in the compound.

\begin{tabular}{rrrrrrrrrr}
\hline Alloy & $m_{\text {spin }}^{\mathrm{X}}$ & $m_{\text {orb }}^{\mathrm{X}}$ & $m_{\text {spin }}^{\mathrm{Y}}$ & $m_{\text {orb }}^{\mathrm{Y}}$ & $m_{\text {spin }}^{\mathrm{Z}}$ & $m_{\text {orb }}^{\mathrm{Z}}$ & $m_{\text {spin }}^{\text {total }}$ & $m_{\text {orb }}^{\text {total }}$ & $m^{\text {total }}$ \\
\hline $\mathrm{FeMnSb}$ & -0.97 & -0.06 & 2.94 & 0.03 & -0.04 & $\sim-0$ & 1.96 & -0.03 & 1.93 \\
$\mathrm{CoMnSb}$ & -0.16 & -0.04 & 3.20 & 0.03 & -0.10 & $\sim-0$ & 2.96 & -0.01 & 2.95 \\
$\mathrm{NiMnSb}$ & 0.25 & 0.02 & 3.72 & 0.03 & -0.07 & $\sim-0$ & 3.95 & 0.04 & 3.99 \\
\hline $\mathrm{Co}_{2} \mathrm{MnAl}$ & 0.75 & 0.01 & 2.60 & 0.01 & -0.09 & $\sim 0$ & 4.00 & 0.04 & 4.04 \\
$\mathrm{Co}_{2} \mathrm{MnSi}$ & 0.99 & 0.03 & 3.02 & 0.02 & -0.08 & $\sim 0$ & 4.93 & 0.08 & 5.00 \\
$\mathrm{Co}_{2} \mathrm{CrAl}$ & 0.70 & 0.01 & 1.64 & 0.01 & -0.08 & $\sim 0$ & 2.97 & 0.03 & 3.00 \\
$\mathrm{Mn}_{2} \mathrm{VAl}$ & -1.40 & -0.03 & 0.79 & -0.01 & 0.01 & 0.01 & -2.00 & -0.07 & -2.07 \\
\hline
\end{tabular}

\%. First one sees that, upon compression, the Fermi level moves in the direction of the conduction band, upon expansion towards the valence band. In both cases, however, the half-metallic character is conserved. In order to explain this behaviour, we first note that the position of Fermi level is determined by the metallic DOS in the majority band. As we believe, the shift of $E_{F}$ is determined by the behaviour of the $\mathrm{Sb} p$-states, in particular by the large extension of these states as compared to the $d$ states. Upon compression the $p$-states are squeezed and hybridize stronger, thus pushing the $d$-states and the Fermi level to higher energies, i.e., towards the minority conduction band. In addition the $\mathrm{Mn} d$ and $\mathrm{Ni}$ or Co $d$ states hybridize stronger, which tends to increase the size of the gap while the $\mathrm{Mn}(\mathrm{Ni}) d$-bandwidth increases, which tends to shrink the gap. As shown in figure 2 the first of the two effects is stronger leading finally to an increase of the gap width. Upon expansion the opposite effects are observed. In the case of NiMnSb and for the experimental lattice constant the gap-width is $\sim 0.4 \mathrm{eV}$. When the lattice is expanded by $2 \%$ the gap shrinks to $0.25 \mathrm{eV}$ and when compressed by $2 \%$ the gap-width is increased to $0.5 \mathrm{eV}$. Similarly in the case of $\mathrm{CoMnSb}$, the gap is $0.8 \mathrm{eV}$ for the experimental lattice constant, $0.65 \mathrm{eV}$ for the $2 \%$ expansion and $0.9 \mathrm{eV}$ for the case of the $2 \%$ compression.

For the full-Heusler alloys the pressure dependence has been recently studied by Picozzi et al. 32 for $\mathrm{Co}_{2} \mathrm{MnSi}, \mathrm{Co}_{2} \mathrm{MnGe}$ and $\mathrm{Co}_{2} \mathrm{MnSn}$, using both the LDA and the somewhat more accurate GGA. The general trends are similar: the minority gap increases with compression, and the Fermi level moves in the direction of the conduction band. For example in the case of $\mathrm{Co}_{2} \mathrm{MnSi}$ the gap-width is $0.81 \mathrm{eV}$ for the theoretical equilibrium lattice constant of $10.565 \AA$. When the lattice constant is compressed to $\sim 10.15 \AA$, the gap-width increases to about $1 \mathrm{eV}$. The calculations show that for the considered changes of the lattice constant of $\pm 2 \%$, half-metallicity is preserved. There can be sizeable changes of the local moments, but the total moment remains constant, since $E_{F}$ stays within the gap.

The results presented in this section up to now concern the homogeneous change of the lattice constant under hydrostatic pressure. Block and collaborators have studied the effect of tetragonalization on the properties of both $\mathrm{NiMnSb}$ and $\mathrm{Co}_{2} \mathrm{CrAl}$ [3]. They found that although tetragonalization largely affected the shape and width of the gap, the systems remained half-metallic for moderate degrees of strain. 


\section{Spin-orbit coupling and orbital magnetism}

The calculations presented up to this point have neglected the spin-orbit coupling (SOC). Intuitively, however, one expects that SOC can be of crucial importance for the half-metallic property: In the presence of SOC, the electron spin is no more a good quantum number, so that the electron eigenfunctions cannot conserve their spin degree of freedom. Spin-up wavefunctions within the half-metallic gap must then have partly spin-down character. As a result, the celebrated half-metallic gap cannot really be $100 \%$ there even at $T \rightarrow 0$. In materials where the SOC is weak, the DOS within the "gap" is expected to be low, and the polarization close to, but not exactly at, $100 \%$ 34. Another result of SOC is the appearance of orbital magnetic moment. This, too, is weak in Heusler alloys with low SOC strength.

The spin polarization $P(E)$ at an energy $E$ (and in particular at $E_{F}$ ) is related to the spin-dependent DOS via the expression

$$
\begin{aligned}
P & =\frac{n_{\uparrow}\left(E_{F}\right)-n_{\downarrow}\left(E_{F}\right)}{n_{\uparrow}\left(E_{F}\right)+n_{\downarrow}\left(E_{F}\right)} \\
& \approx 1-2 n_{\downarrow}\left(E_{F}\right) / n_{\uparrow}\left(E_{F}\right) \quad \text { for small } n_{\downarrow} / n_{\uparrow} .
\end{aligned}
$$

The spin-orbit coupling connecting the two spin channels reads, in terms of the Pauli matrices $\vec{\sigma}$ and the orbital momentum $\vec{L}$ :

$$
V_{\mathrm{so}}(r)=\frac{1}{2 m^{2} c^{2}} \frac{\hbar}{2} \frac{1}{r} \frac{d V}{d r} \vec{L} \cdot \vec{\sigma}=\left(\begin{array}{ll}
V_{\mathrm{so}}^{\uparrow \uparrow} & V_{\mathrm{so}}^{\uparrow \downarrow} \\
V_{\mathrm{so}}^{\downarrow \uparrow} & V_{\mathrm{so}}^{\downarrow \downarrow}
\end{array}\right) .
$$

Here, $V(r)$ is the unperturbed one-electron potential at an atomic site, and is assumed to be spherically symmetric. Deviations from the spherical symmetry arise only close to the interstitial region between atoms, where the contribution to the spin-orbit interaction is anyhow small. The $2 \times 2$ matrix is the perturbation expressed in spinor basis, demonstrating the non-diagonal terms $V_{\text {so }}^{\uparrow \downarrow}$ and $V_{\text {so }}^{\downarrow \uparrow}$ which are responsible for spin-flip processes; $\uparrow$ and $\downarrow$ denote the spin up and spin down direction. Within perturbation theory one can show that the spin-down DOS within the gap is a weak reflection of the spin-up DOS, depending quadratically on the spin-orbit coupling strength: $n_{\downarrow} \sim n_{\uparrow}\left(V_{\mathrm{so}}^{\uparrow \downarrow}\right)^{2}$ [34. Moreover it can be shown that, close to the gap edges, there is an enhancement of the spin-flip elements.

In reference 34, $P\left(E_{F}\right)$ was calculated for a number of half-Heusler alloys and other half-metals. The calculations were done within density-functional theory, utilising the KKR Green function method [19]. The approach was fully relativistic, solving the Dirac equation rather than treating the spin-orbit coupling as a perturbation. Nevertheless the qualitative behaviour which was described above was revealed. Table 2 summarizes the results for $P\left(E_{F}\right)$ and for the polarization in the middle of the gap, $P\left(E_{M}\right)$, for some half-Heusler alloys. The trend is that alloys which include heavier elements show a lower spin polarization. This becomes most evident by inspection of the values of $P\left(E_{M}\right)$, since for some of the alloys the Fermi level approaches or enters the valence band, so that $P\left(E_{F}\right)$ does not always reflect the SOC strength. In particular, NiMnSb shows a high value for $P\left(E_{M}\right)(99.3 \%)$, which decreases when we substitute the $3 d$ element $\mathrm{Ni}$ with the heavier, $4 d$ element, $\mathrm{Pd}$, and even more so when we substitute it with the even heavier, $5 d$ element, Pt. This effect is only expected, since it is well-known that heavier elements show in general a stronger SOC due to the term $d V / d r$ in Eq. 4 As a conclusion, the spin-orbit coupling reduces $P\left(E_{F}\right)$, but the resulting values are still high. Nevertheless, we point out that calculations on half-metallic ferromagnets containing heavy elements (e.g., 
Table 2. Calculated spin polarisation $P$ at the Fermi level $\left(E_{F}\right)$ and in the middle of the spin-down gap $\left(E_{M}\right)$, and ratio of spin-down/spin-up DOS in the middle of the gap $\left(n_{\downarrow} / n_{\uparrow}\right)\left(E_{M}\right)$, for various half-Heusler alloys. The alloys PdMnSb and PtMnSb present a spin-down gap, but are not half-metallic, as $E_{F}$ is slightly below the gap.

\begin{tabular}{cccc}
\hline Compound & $P\left(E_{F}\right)$ & $P\left(E_{M}\right)$ & $\left(n_{\downarrow} / n_{\uparrow}\right)\left(E_{M}\right)$ \\
\hline CoMnSb & $99.0 \%$ & $99.5 \%$ & $0.25 \%$ \\
FeMnSb & $99.3 \%$ & $99.4 \%$ & $0.30 \%$ \\
NiMnSb & $99.3 \%$ & $99.3 \%$ & $0.35 \%$ \\
PdMnSb & $40.0 \%$ & $98.5 \%$ & $0.75 \%$ \\
PtMnSb & $66.5 \%$ & $94.5 \%$ & $2.70 \%$ \\
\hline
\end{tabular}

lanthanides) should take into account the spin-orbit coupling for a reliable quantitative result.

The orbital moments in Heusler alloys are expected to be small. This is a result of the cubic symmetry, of the fact that the magnetic transition elements here are relatively light ( $3 d$ series) and of the metallic nature of the electronic states.

Orbital moments were calculated by Galanakis et al. for half-Heusler compounds 35, by Picozzi et al. 32 for $\mathrm{Co}_{2} \mathrm{MnSi},-\mathrm{Ge}$, and -Sn, and more systematically by Galanakis 36. Some of the latter results are summarized in table 1 As expected, the values of the orbital moments $m_{\text {orb }}$ (calculated within the LSDA) are small. It is known that the LSDA can underestimate $m_{\text {orb }}$ by up to $50 \%$, but the trends are considered reliable. The highest orbital moment, almost $0.1 \mu_{B}$, appears at the Ir and Mn atoms in IrMnSb, but with opposite signs for the two atoms, so that the total $m_{\text {orb }}$ is close to zero (note that also the Ir spin moment is opposite to the Mn spin moment; for Ir in this compound we obtain a ratio of $m_{\text {orb }} / m_{\text {spin }} \simeq 1 / 2$ ).

\section{Doping, disorder and defects}

Before starting our discussion on doping, disorder and defects in Heusler alloys, we should note that, as shown by Larson et al [37, the ordered structure of Heusler alloys is the one with the minimum energy and thus the more stable.

Disorder in NiMnSb had been studied initially by Orgassa and collaborators [38]. They took into account three different types of disorder: i) intermixing of $\mathrm{Ni}$ and $\mathrm{Mn}$ atoms, ii) migration of $\mathrm{Ni}$ and $\mathrm{Mn}$ atoms at the empty site (the one occupied by the second high-valent transition metal atom in the full Heusler alloys), and iii) migration of $\mathrm{Mn}$ and $\mathrm{Sb}$ atom to the empty site. They found that $5 \%$ of disorder was enough so that the impurity states destroy the minority-spin gap. This work was recently extended by Alling et al. 39 covering all possible kinds of interstitial, vacancy and atomic-swap defects in NiMnSb in the diluted limit. They have shown that defects span a large area of energy formation values, from 0.2 to $14.4 \mathrm{eV}$. More interestingly defects with formation energies less than $4 \mathrm{eV}$, which are also the most likely to occur, do not destroy the half-metallicity although they severely affect the width and shape of the gap.

The formation of defects in full Heusler alloys and more precisely in $\mathrm{Co}_{2} \mathrm{MnGe}$ and $\mathrm{Co}_{2} \mathrm{MnSi}$ has been studied by Picozzi et al [40. They found that the Mn antisite have the lowest formation energy and they retain half-metallicity contrary to the Co ones. The Mn-Si and Mn-Co atomic swaps were found to have very large formation 
energies and thus are unlikely to occur. But these results cannot be generalized to all Heusler alloys. As shown by Miura et al in the case of $\mathrm{Co}_{2} \mathrm{CrAl}$ the $\mathrm{Cr}-\mathrm{Al}$ swaps have low formation energy and the system prefers energetically the $B 2$ structure where the $\mathrm{Cr}$ and $\mathrm{Al}$ atoms are completely disordered 41. Substituting $\mathrm{Fe}$ for $\mathrm{Cr}$ restores the order since the $\mathrm{Fe}-\mathrm{Al}$ atomic swaps have very high formation energy and are unlikely to occur.

Galanakis et al studied the effect of doping and disorder on the properties of the half-metallic full Heusler alloys: $\mathrm{Co}_{2} \mathrm{MnSi}, \mathrm{Co}_{2} \mathrm{MnGe}$ and $\mathrm{Co}_{2} \mathrm{MnSn}$ [42]. To simulate the doping by electrons we substituted Mn by Fe while to simulate the doping of the alloys with holes we substituted Mn by Cr. We studied substitution of 5\%, $10 \%$ and $20 \%$ of the Mn atoms. Doping the perfectly ordered alloys with either Fe or Cr induces a smoothening of the DOS. Cr-doping has only marginal effects to the gap. Its width is narrower with respect to the perfect compounds but overall the compounds retain their half-metallicity. In the case of Fe-doping the situation is more complex. Adding electrons to the system means that, in order to retain the perfect half-metallicity, these electrons should occupy majority antibonding levels at higher energies. This is energetically not very favourable and in the case of $\mathrm{Co}_{2} \mathrm{MnSi}$ and for $20 \% \mathrm{Fe}$ doping the Fermi level is no more exactly in the gap but slightly above it. In general the total spin moment follows the Slater-Pauling behaviour with only slight deviations for the $\mathrm{Co}_{2} \mathrm{MnSi}$ compound with for Fe-doping.

Finally we discuss the effect of disorder between the Mn and the $s p$ atoms in $\mathrm{Co}_{2} \mathrm{MnSi}, \mathrm{Co}_{2} \mathrm{MnGe}$ and $\mathrm{Co}_{2} \mathrm{MnSn}$ [2]. If one inspects the atom-resolved DOS, the gap showing at the DOS of the Mn and $s p$ atoms is wider than the gap showing at the DOS of the Co atoms. This is because the states around the "total" gap are of Co-character only, as discussed in section 2 Intermixing $\mathrm{Mn}$ and the $s p$ atoms changes the symmetry of the Co sites and in this way new states in the gap can be induced, affecting the half-metallic character. Substituting $\mathrm{Mn}$ by $\mathrm{Si}$ in $\mathrm{Co}_{2} \mathrm{MnSi}$ induces states just at the high edge of the gap while substituting Si by Mn pushes the unoccupied minority states even higher in energy and the gap becomes wider. Substituting 5\%, $10 \%$ or $20 \%$ of the Mn atoms by Si results in a decrease of $0.15,0.30$ and 0.60 of the total number of valence electrons in the cell, while the inverse procedure results to a similar increase of the mean value of the number of valence electrons. The situation is analogous in $\mathrm{Co}_{2} \mathrm{MnGe}$ and $\mathrm{Co}_{2} \mathrm{MnSn}$. The compounds containing $\mathrm{Si}$ and Ge show a perfect Slater-Pauling behaviour (as a function of the substitution fraction $x$ ) while the $\mathrm{Co}_{2} \mathrm{Mn}_{1+x} \mathrm{Sn}_{1-x}$ deviate from the ideal values of the total spin moment.

\section{Quaternary Heusler alloys}

We proceed with a discussion on the quaternary Heusler alloys [41, 43. In the these compounds, one of the four sites is occupied by two different kinds of neighbouring elements, like $\mathrm{Co}_{2}\left[\mathrm{Cr}_{1-x} \mathrm{Mn}_{x}\right] \mathrm{Al}$, where the $\mathrm{Y}$ site is occupied by $\mathrm{Cr}$ or $\mathrm{Mn}$ atoms. For all calculations we assumed that the lattice constant varies linearly with the concentration $x$ which has been verified for several quaternary alloys [3].

In reference [43], calculations are reported on several quaternary alloys taking into account several possible combinations of chemical elements and considering a concentration increment of 0.1 . The results are resumed in figure B In the first case, two different low-valent transition metal atoms can occupy the $\mathrm{Y}$ site, as in $\mathrm{Co}_{2}\left[\mathrm{Cr}_{1-x} \mathrm{Mn}_{x}\right] \mathrm{Al}$. The total spin moment varies linearly between the $3 \mu_{B}$ of $\mathrm{Co}_{2} \mathrm{CrAl}$ and the $4 \mu_{B}$ of $\mathrm{Co}_{2} \mathrm{MnAl}$. In the case of $\mathrm{Co}_{2}\left[\mathrm{Cr}_{1-x} \mathrm{Fe}_{x}\right] \mathrm{Al}$ and $\mathrm{Co}_{2}\left[\mathrm{Mn}_{1-x} \mathrm{Fe}_{x}\right] \mathrm{Al}$, 


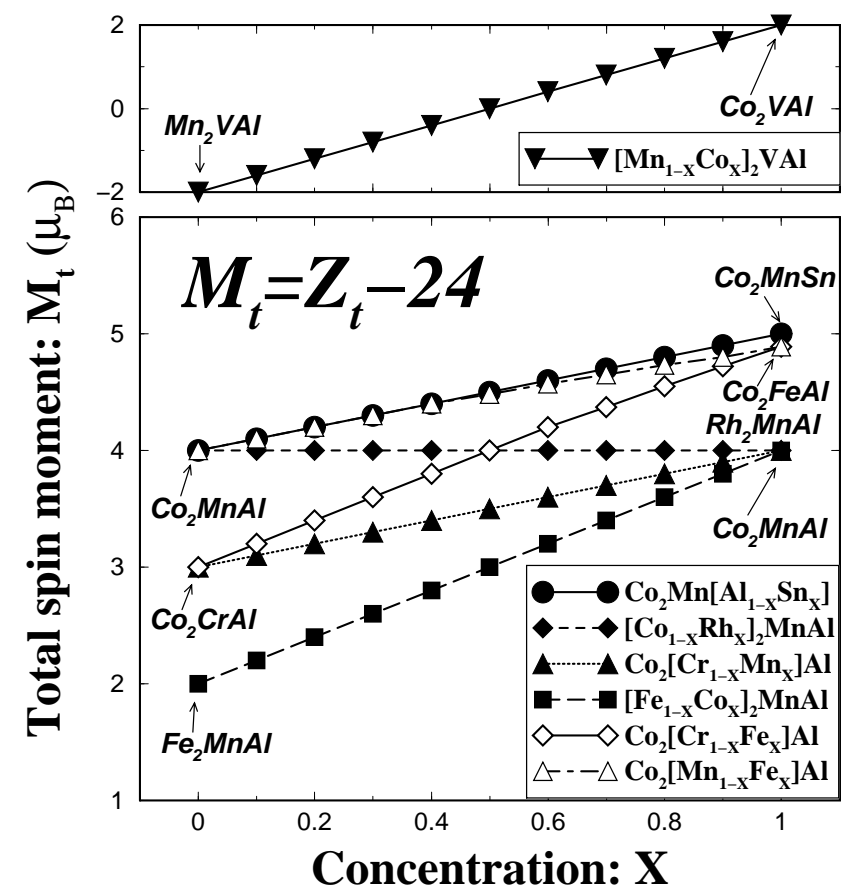

Figure 3. Calculated total spin moment $M_{t}$ in $\mu_{B}$ for a variety of compounds as a function of the concentration $x(x=0,0.1,0.2, \ldots, 0.9,1)$. We assumed that the lattice constant varies linearly with the concentration $x$. With solid lines the cases obeying the rule $M_{t}=Z_{t}-24$ are shown where $Z_{t}$ and $M_{t}$ are the average total number of valence electrons and the average total moment.

and up to around $x=0.6$ the total spin moment shows a Slater-Pauling behaviour but for larger concentrations $M_{t}$ deviates slightly; $\mathrm{Co}_{2} \mathrm{FeAl}$, corresponding to $x=1$, has a non-integer moment. Similar results were also obtained in reference 44]. This behaviour is clearly seen in figure 3 when we compare the lines for the $\mathrm{Co}_{2}\left[\mathrm{Mn}_{1-x} \mathrm{Fe}_{x}\right] \mathrm{Al}$ and $\mathrm{Co}_{2} \mathrm{Mn}\left[\mathrm{Al}_{1-x} \mathrm{Sn}_{x}\right]$ compounds; the latter family follows the $\mathrm{SP}$ behaviour. The latter case brings us to the set of compounds where $s p$ elements are mixed, and these compounds also obey the rule for the total spin moments.

The third case is to mix the higher valent transition metal atoms, as in $\left[\mathrm{Fe}_{1-x} \mathrm{Co}_{x}\right]_{2} \mathrm{MnAl}$ and $\left[\mathrm{Rh}_{1-x} \mathrm{Co}_{x}\right]_{2} \mathrm{MnAl}$ alloys. In $\left[\mathrm{Fe}_{1-x} \mathrm{Co}_{x}\right]_{2} \mathrm{MnAl}$ the total spin moment varies linearly between the $2 \mu_{B}$ of $\mathrm{Fe}_{2} \mathrm{MnAl}$ and $4 \mu_{B}$ of $\mathrm{Co}_{2} \mathrm{MnAl}$. As for $\left[\mathrm{Rh}_{1-x} \mathrm{Co}_{x}\right]_{2} \mathrm{MnAl}$, we observe that $\mathrm{Rh}$ is isoelectronic to $\mathrm{Co}$, thus we find a constant integer value of $4 \mu_{B}$ for all concentrations.

Finally, we discuss the special case of $\mathrm{Mn}_{2} \mathrm{VAl}$, which has less than 24 electrons and a total spin moment of $-2 \mu_{B}$. Here, mixing $\mathrm{Mn}$ and Co gives a family of compounds $\left(\mathrm{Mn}_{1-x} \mathrm{Co}_{x}\right)_{2} \mathrm{VAl}$, where the total spin moment varies linearly between $-2 \mu_{B}$ and $2 \mu_{B}$. Interestingly, for $x=0.5$ we get the case of a compound without macroscopic magnetization, consisting of magnetic atoms (as in the case of an antiferromagnet). Thus all the compounds obey the rule $M_{t}=Z_{t}-24$, showing the Slater-Pauling behaviour regardless of the origin of the extra charge.

As a rule of thumb, we expect that for two half-metallic alloys like XYZ and 
Properties of Heusler alloys calculated from first-principles

Table 3. Half metallic lattice parameters and spin magnetic moments (in $\mu_{B}$ ) of $\mathrm{Mn}_{2} \mathrm{VZ}(\mathrm{Z}=\mathrm{Al}, \mathrm{Si}, \mathrm{Ge}, \mathrm{Sn})$.

\begin{tabular}{lccccc}
\hline Compound & $a(\AA)$ & $m_{\mathrm{Mn}}$ & $m_{\mathrm{V}}$ & $m_{\mathrm{Z}}$ & $m_{\text {Cell }}$ \\
\hline $\mathrm{Mn}_{2} \mathrm{VAl}$ & 5.987 & -1.510 & 0.967 & 0.053 & -2.00 \\
& 6.117 & -1.655 & 1.233 & 0.076 & -2.00 \\
\hline $\mathrm{Mn}_{2} \mathrm{VSi}$ & 6.06 & -0.863 & 0.675 & 0.052 & -1.00 \\
& 6.29 & -1.092 & 1.105 & 0.078 & -1.00 \\
\hline $\mathrm{Mn}_{2} \mathrm{VGe}$ & 6.18 & -0.976 & 0.905 & 0.048 & -1.00 \\
& 6.27 & -1.082 & 1.111 & 0.054 & -1.00 \\
\hline $\mathrm{Mn}_{2} \mathrm{VSn}$ & 6.25 & -0.980 & 0.922 & 0.038 & -1.00 \\
& 6.31 & -1.050 & 1.059 & 0.041 & -1.00 \\
\hline
\end{tabular}

$\mathrm{X}^{\prime} \mathrm{YZ}$ (or $\mathrm{XY}^{\prime} \mathrm{Z}$ or $\mathrm{XYZ}$ ), which both lie on the Slater-Pauling curve, also the mixtures like $\mathrm{X}_{1-x} \mathrm{X}_{x}^{\prime} \mathrm{YZ}$ lie on the Slater Pauling curve, with an average moment of $\left\langle M_{t}\right\rangle=(1-x) M_{t}^{\mathrm{XYZ}}+x M_{t}^{\mathrm{X}^{\prime} \mathrm{YZ}}$. However, it is not guaranteed that these intermediate structures are stable, especially if the parent compounds are not neighbours on the Slater-Pauling curve.

\section{Half-metallic ferrimagnets}

The ideal case for realistic applications would be a half-metallic antiferromagnet rather than the half-metallic ferromagnets. In such a compound the majority- and minority-spin densities of states are not connected by a symmetry transformation, as for common antiferromagnets and the material is better described as a fully compensated ferrimagnet, having a net magnetic moment that is, due to the halfmetallic character, precisely equal to zero. Such a material would have the advantage that due to the absence of a total magnetic moment it would not give rise to stray fields. Van Leuken and de Groot have suggested a possible route towards a halfmetallic antiferromagnet starting from the semiconducting $C 1_{b}$-type compound $\mathrm{FeVSb}$ 45. Due to the difficulties to construct such a material, it would be useful to study also the half-metallic ferrimagnetic materials, like FeMnSb [46] or the $\mathrm{Mn}_{2} \mathrm{VZ}$ [4] compounds where the $\mathrm{Mn}$ and $\mathrm{Fe}$ or V spin moments are anti-parallel.

In reference [48] we studied the possibility of appearance of half-metallicity in the case of the full-Heusler compounds $\mathrm{Mn}_{2} \mathrm{VZ}$ where $\mathrm{Z}$ is an $s p$ atom belonging to the IIIB or IVB column of the periodic table. Total energy calculations have shown that, when $\mathrm{Z}$ is $\mathrm{Al}, \mathrm{Ga}$, In or $\mathrm{Sn}$, the compounds are ferrimagnetic, while the compounds containing $\mathrm{Si}$ and $\mathrm{Ge}$ are non-magnetic at the equilibrium lattice constant. A small expansion of the lattice leads to the emergence of ferrimagnetism also in these compounds.

Although all compounds are not half-metallic at their equilibrium lattice constant, small expansion of the lattice pushes the Fermi level within the gap which is now situated in the majority-spin band contrary to all other full-Heusler alloys. In table 3 we have gathered the atom-resolved and total spin moments for some of the compounds under study, for the largest and smallest lattice parameters for which half-metallicity is present. The total spin moment is $-2 \mu_{B}$ for the $\mathrm{Mn}_{2} \mathrm{VAl}$ (Ga or In) compounds which have 22 valence electrons per unit cell and $-1 \mu_{B}$ for the $\mathrm{Mn}_{2} \mathrm{VSi}(\mathrm{Ge}$ or $\mathrm{Sn}$ ) compounds with 23 valence electrons (the negative sign of $M_{t}$ is according to the convention set by equation 21. Thus these compounds follow the Slater-Pauling behaviour and the "rule of 24". The lighter the element and the smaller the number of valence electrons, 
the wider is the gap and the more stable is the half-metallicity with respect to the variation of the lattice constant.

\section{Effect of temperature on the spin polarization}

The discussion so-far has been focused on the magnetic ground state of half-metallic Heusler alloys. At elevated temperatures $T$ the spin polarization $P\left(E_{F} ; T\right)$ must clearly be reduced, and at the Curie temperature $T_{C}$ it must vanish together with the average magnetization. An qualitative and quantitative understanding of this effect is interesting from the point of view of basic science, but is also required in order to predict the applicability of half-metals in realistic devices.

A theoretical study of the temperature dependence of $P\left(E_{F}\right)$ requires knowledge of the response of the spectral function (density of states) to an increase of the temperature. This is a two-step process. Firstly, one needs to find the response of the magnetic excitations to temperature. Secondly, the spectral function must be found in the presence of the excitations. Both steps are not trivial, thus suitable approximations are needed. To our knowledge, there have been three different approaches to the subject for the case of half-metallic ferromagnets: a tight-binding approach [49, an approach based on constrained density-functional theory combined with the concept of a disordered local moment [50], and an approach based on dynamical mean-field theory (DMFT) 51, 52.

Skomski and Dowben [49] consider a tight-binding model of a half-metallic ferromagnet. Magnetic excitations, in the form of magnons, will cause a rotation of the spin quantization axis, in general different at each atom $i$, given by a unitary transformation $U\left(\theta_{i}, \phi_{i}\right)$. This causes a projection of spin-up states of one atom to spin-down states of another. They arrive at a crude estimate of this spin-mixing contribution to the spin-down DOS: $n_{\downarrow} \simeq n_{\uparrow}\left(M_{0}-M(T)\right) /\left(M_{0}+M(T)\right)$, in terms of the spin-up DOS $n_{\uparrow}$, the magnetization $M_{0}$ at $T=0$, and at temperature $T$, $M(T)$. This gives an estimate for the polarization $P(T) \simeq M(T) / M_{0}$. This picture is appealing because of its simplicity, and should be correct when the magnetic excitations are predominantly of the Heisenberg type and have a long wavelength, i.e., at low temperatures. Then, the local spin quantization axis is unchanged over large distances (of the order of a fraction of the magnon wavelength), and, at each instant, the material can be considered locally half-metallic; the reduction of $P$ comes about from the angle between the local spin quantization axes and the global axis corresponding to the average magnetic moment.

However, as is argued by Ležaić et al. [50], at higher temperatures the wavelength of the magnetic excitations can become smaller and affect the local electronic structure. The hybridization between atomic wavefunctions is crucial for the gap, and the hybridization strength can be altered if the local spin axes of neighbouring atoms are different. Therefore, the spectral function should be in principle recalculated self-consistently in the presence of the excitations. Since the characteristic times of magnetic excitations are much longer than the electron hopping times, the adiabatic approximation within density-functional theory can be employed: an external constraint is used to force a non-collinear state, representing the magnon; under this constraint, the electronic structure is calculated self-consistently within density-functional theory.

Such an approach was applied in reference [50, with the conclusion that a non-collinear configuration can affect the hybridization strongly enough that the 
polarization collapses. In particular, it was found for $\mathrm{NiMnSb}$ that, under the constraint of a spin spiral, the gap does not close easily, but the minority-spin $d$ bands around the gap move in such a way that they cross the Fermi level. Because the $d$ bands have a high spectral weight, $n_{\downarrow}\left(E_{F}\right)$ increases abruptly when these reach $E_{F}$, and the polarization collapses. The effect was quantified by using the disordered local moment approach. Within this method, the magnetic state at $T>0$ is approximated by a state of the form $\mathrm{Ni}\left(\mathrm{Mn}_{1-x}^{\uparrow} \mathrm{Mn}_{x}^{\downarrow} S b\right.$, i.e., the spin is flipped a fraction $x$ of the $\mathrm{Mn}$ atoms in a random way, representing a disordered moment at the Mn site. For $x=0$ the ferromagnetic ground state is recovered, while $=0.5$ corresponds to $T_{C}$. The electronic structure of this disordered state was found within the CPA and the KKR Green function method. In this way, $P$ can be calculated as a function of $M$, and, if $M(T)$ is independentlu known, one can find $P(T)$. The results show a collapse of the polarization at about $0.4 T_{C}$, which corresponds to room temperature.

Chioncel et al. 51, 53] have considered the effect of the dynamic electron-magnon interaction, resulting from electron correlations. According to this picture, there exist excitations within the (spin-down) half-metallic gap as superpositions of spin-up electron states and spin-down electron states with magnons [54. These excitations, also called non-quasiparticle states, produce at $T=0$ a spectral weight at energies just above $E_{F}$ (or just below $E_{F}$ if the gap is among the majority-spin states). For $T>0$, however, spin-down spectral weight appears also at $E_{F}$, and the polarization is reduced. This correlation-induced effect depends on the value of the on-site coulomb interaction $U$, and can be captured within the DMFT. For the Heusler alloy FeMnSb, calculations within the LDA+DMFT reported in [53] show that, at $T=300 K, P \simeq 0.9$ if $U=2 \mathrm{eV}$ and $P \simeq 0.6$ if $U=4 \mathrm{eV}$.

Unfortunately, there are not many experimental results concerning the temperature dependence of the polarization in Heusler alloys. One indirect result is provided in reference 10, where tunneling magnetoresistance (TMR) data are compared from junctions containing a $\mathrm{Co}_{2} \mathrm{MnSi}$ Heusler alloy as a first electrode with data from junctions with $\mathrm{Co}_{70} \mathrm{Fe}_{30}$ or $\mathrm{Ni}_{80} \mathrm{Fe}_{20}$ (the second electrode is in all cases $\mathrm{Co}_{70} \mathrm{Fe}_{30}$, and the barrier is $\mathrm{AlO}_{x}$ ). It is shown that, at low temperatures, the $\mathrm{Co}_{2} \mathrm{MnSi}$-based junction exhibits an $85 \%$ TMR ratio (relative change of resistance due to a change of alignment of the magnetic moments of the leads), while the $\mathrm{Co}_{70} \mathrm{Fe}_{30^{-}}$and $\mathrm{Ni}_{80} \mathrm{Fe}_{20}$-based junctions exhibit $65 \%$ and $70 \%$ TMR ratio respectively. At higher temperatures (above $150 \mathrm{~K}$ ) the situation is reversed, with the $\mathrm{Co}_{2} \mathrm{MnSi}$ based junction showing a lower TMR ratio $(30 \%$ at $300 \mathrm{~K}$ ) than the other two $(45 \%$ at $300 \mathrm{~K}$ ). This evidence suggests that, at low $T, \mathrm{Co}_{2} \mathrm{MnSi}$ has a higher spin polarization than $\mathrm{Co}_{70} \mathrm{Fe}_{30}$ or $\mathrm{Ni}_{80} \mathrm{Fe}_{20}$, but at high $T$, the polarization of the Heusler alloy drops more rapidly. We note here that $\mathrm{Co}_{2} \mathrm{MnSi}$ has a high Curie temperature of $985 \mathrm{~K}$.

More recently, Sakuraba and collaborators 12 managed to grow a $\mathrm{Co}_{2} \mathrm{MnSi} / \mathrm{Al}-$ $\mathrm{O} / \mathrm{Co}_{2} \mathrm{MnSi}$ tunnel junction. At low temperatures $(2 \mathrm{~K})$, an extremely high TMR ratio of $570 \%$ was reported, while with increasing temperature the TMR dropped rapidly, reaching about $67 \%$ at $300 \mathrm{~K}$. On the other hand, a $\mathrm{Co}_{2} \mathrm{MnSi} / \mathrm{Al}-\mathrm{O} / \mathrm{Co}_{75} \mathrm{Fe}_{25}$ junction showed a less pronounced decrease of TMR from $160 \%$ at $2 \mathrm{~K}$ to $70 \%$ at $300 \mathrm{~K}$, while a junction $\mathrm{Co}_{75} \mathrm{Fe}_{25} / \mathrm{Al}-\mathrm{O} / \mathrm{Co}_{75} \mathrm{Fe}_{25}$ showed a less drastic decrease of TMR, approximately from $60 \%$ to $30 \%$. The authors of reference [12 propose an explanation in terms of spin-flip processes due to magnetic impurities at the interface $\mathrm{Co}_{2} \mathrm{MnSi} / \mathrm{Al}-\mathrm{O}$. Alternatively, it is possible that there is a rapid loss of half-metallicity with increasing temperature, causing a decrease of $P$ much faster than $M$. 
Properties of Heusler alloys calculated from first-principles

\section{Summary and Outlook}

In this review we have given an introduction to the electronic structure and the resulting magnetic properties of half-metallic Heusler alloys, which represent interesting hybrids between metallic ferromagnets and semiconductors.

First, we reviewed a few recent results on the electronic properties of these alloys. The origin of the gap in these half-metallic alloys and its connection to the magnetic properties are well understood. The gap in half-Heuslers arises between the bonding and antibonding $d$-hybrids created by the transition metal atoms (e.g. Ni and $\mathrm{Mn}$ in $\mathrm{NiMnSb}$ ). In full-Heusler compounds, like $\mathrm{Co}_{2} \mathrm{MnSi}$, there is the extra complication of the appearance of Co $d$-states which do not hybridize with the Mn states. There are exactly 9 occupied minority-spin states for the half-Heuslers and 12 for the fullHeuslers; thus the total spin moment follows a Slater-Pauling behaviour for both families. Moderate changes of the lattice parameter shift the Fermi level, but overall do not affect half-metallicity. Spin-orbit coupling also induces states within the gap but the alloys keep a very high degree of spin-polarization at the Fermi level. Orbital moments are small as expected for typical intermetallic ferromagnets.

We discussed the effect of substitutional doping, disorder and defects on the properties of these alloys. In many cases moderate degrees of disorder and doping do not alter half-metallicity. Also defects with low formation energies, which are the most likely to occur, keep the half-metallic character of the compounds. Quaternary Heusler alloys are a special case of full-Heusler alloys where one site is occupied randomly by two chemical elements. These alloys, when the two extreme normal compounds are half-metallic, follow the Slater-Pauling behaviour keeping the halfmetallicity of the normal Heusler alloys. Finally we discuss the appearance of halfmetallic ferrimagnetism in $\mathrm{Mn}_{2} \mathrm{VZ}$ ( $\mathrm{Z}$ and $s p$ element).

The results summarized above contribute in the idea that half-metallic ferromagnets are feasible experimentally since all studied effects keep an almost $100 \%$ degree of spin-polarization at the Fermi. However as discussed in the last section temperature effects quickly destroy the half-metallicity and the alloys transit to a normal metal. Moreover, surfaces/interfaces of Heusler alloys are known from first principles calculations to show strong surface/interface states which can severely reduce the degree of spin-polarization [16] 17. A theoretical analysis [55] shows that interface states at a half-metal/semiconductor interface can reduce the tunneling magnetoresistance ratio significantly.

\section{Acknowledgments}

The authors would like to thank their collaborators in the research projects presented here: P.H. Dederichs, N. Papanikolaou, R. Zeller, H. Ebert, V. Popescu, K. Özdog̃an, E. Şaşıỡlu, B. Aktaş, L.M. Sandratskii, P. Bruno, M. Ležaić, G. Bihlmayer, and S. Blügel.

\section{References}

[1] de Groot R A, Mueller F M, van Engen P G and Buschow K H J 1983 Phys. Rev. Lett. 502024

[2] Žutić I, Fabian J and Das Sarma A 2004 Rev. Mod. Phys. 76323

[3] Webster P J and Ziebeck K R A, in Alloys and Compounds of d-Elements with Main Group Elements. Part 2., edited by H.R.J. Wijn, Landolt-Börnstein, New Series, Group III, Vol. 19,Pt.c (Springer-Verlag, Berlin), 1988, pp. 75-184 
[4] Ziebeck K R A and Neumann K -U, in Magnetic Properties of Metals, edited by H. R. J. Wijn, Landolt-Börnstein, New Series, Group III, Vol. 32/c (Springer, Berlin), 2001, pp. 64-414

[5] Soulen Jr R J, Byers J M, Osofsky M S, Nadgorny B, Ambrose T, Cheng S F, Broussard P R, Tanaka C T, Nowak J, Moodera J S, Barry A and Coey J M D 1998 Science 28285

[6] Kilian K A and Victora R H 2000 J. Appl. Phys. 87, 7064

[7] Tanaka C T, Nowak J, and Moodera J S 1999 J. Appl. Phys. 86, 6239

[8] Caballero J A, Park Y D, Childress J R, Bass J, Chiang W-C, Reilly A C, Pratt Jr. W P, and Petroff F 1998 J. Vac. Sci. Technol. A 16, 1801 ; Hordequin C, Nozières J P, and Pierre J 1998 J. Magn. Magn. Mater. 183, 225

[9] Bergmann A, Grabis J, Toperverg B P, Leiner V, Wolff M, Zabel H, and Westerholt K 2005 Phys. Rev. B 72 214403; Grabis J, Bergmann A, Nefedov A, Westerholt K, and Zabel J 2005 Phys. Rev. B 72 024437; idem 2005 Phys. Rev. B 72024438

[10] Kämmerer S, Thomas A, Hütten A, and Reiss G 2004 Appl. Phys. Lett. 8579

[11] Schmalhorst J, Kämmerer S, Sacher M, Reiss G, Hütten A, and Scholl A 2004 Phys. Rev. B 70 024426

[12] Sakuraba Y, Hattori M, Oogane M, Ando Y, Kato H, Sakuma A, Miyazaki T, and Kubota H 2006 Appl. Phys. Lett. 88 192508; Sakuraba Y, Nakata J, Oogane M, Kubota H, Ando Y, Sakuma A, and Miyazaki T 2005 Japan. J. Appl. Phys. 44 L1100

[13] Dong X Y, Adelmann C, Xie J Q, Palmström C J, Lou X, Strand J, Crowell P A, Barnes J P, and Petford-Long A K 2005 Appl. Phys. Lett. 86102107

[14] Galanakis, I, Mavropoulos Ph, and Dederichs P H 2006 J. Phys. D: Appl. Phys. 39765

[15] Galanakis I and Dederichs P H (ed) 2005 Half-metallic alloys: fundamentals and applications (Lecture notes in Physics vol 676) (Berlin Heidelberg: Springer)

[16] Galanakis I, Ležaić M, Bihlmayer G, and Blügel S 2005 Phys. Rev. B 7171 214431; Ležaić M, Galanakis I, Bihlmayer G, and Blügel S 2005 J. Phys.: Condens. Matter 17 3121; Galanakis I 2005 J. Magn. Magn. Mater. 288 411; Galanakis I 2004 J. Phys.: Condens. Matter 16 8007; Galanakis I 2002 J. Phys.: Condens. Matter 146329

[17] Jenkins S J 2004 Phys. Rev. B 70 245401; Jenkins S J and King D A 2002 Surf. Sci 501 L185; idem 2001 Surf. Sci 494 L793

[18] Papanikolaou N, Zeller R and Dederichs P H 2002 J. Phys.: Condens. Matter 142799

[19] The SPR-TB-KKR package, H. Ebert and R. Zeller, http://olymp.cup.uni-muenchen.de/ak/ebert/SPR-TB-KKR/

[20] Koepernik K and Eschrig H 1999 Phys. Rev. 59 3174; Koepernik K, Velicky B, Hayn R and Eschrig H 1998 Phys. Rev. B 586944

[21] The KKR-CPA-LDA package AkaiKKR(Machikanekama) is available from http://sham.phys.sci.osaka-u.ac.jp/ kkr/

[22] Galanakis I, Dederichs P H and Papanikolaou N 2002 Phys. Rev. B 66134428

[23] Galanakis I, Dederichs P H, and Papanikolaou N 2002 Phys. Rev. B 66174429

[24] Ishida S, Fujii S, Kashiwagi S and Asano S 1995 J. Phys. Soc. Jpn. 64 2152; Fujii S, Ishida S and Asano S 1995 J. Phys. Soc. Jpn. 64 185; Zhang M, Liu Z H, Hu H N, Liu G D, Cui Y T, Wu G H, Brc̈k R, de Boer F R, and Li Y X 2004 J. Appl. Phys. 95 7219; Zhang M, Dai X, Hu H, Liu G, Cui Y, Liu Z, Chen J, Wang J, and Wu G 2003 J. Phys.: Condens. Matter 157891

[25] Plogmann S, Schlathölter T, Braun J, Neumann M, Yarmoshenko Yu M, Yablonskikh M V, Shreder E I, Kurmaev E Z, Wrona A and Ślebarski A 1999 Phys. Rev. 606428

[26] Nanda B R K and Dasgupta I 2003 J. Phys.: Condens. Matter 15, 7307

[27] Jung D, Koo H J and Whangbo M J 2000 J. Mol. Struct. (Theochem) 527113

[28] Kübler J 1984 Physica B \& C 127257

[29] Kandpal H M, Fecher G H, Felser C, and Schönhense G 2006 Phys. Rev. B 73094422

[30] Şaşıŏlu E, Sandratskii L M, Bruno P, and Galanakis I 2005 Phys. Rev. 72184415

[31] Kurtulus Y, Dronskowski R, Samolyuk G D, and Antropov V P 2005 Phys. Rev. B 71014425

[32] Picozzi S, Continenza A and Freeman A J 2002 Phys. Rev. B 66094421

[33] Block T, Carey M J, Gurney B A, and Jepsen O 2004 Phys. Rev. B 70205114

[34] Mavropoulos Ph, Sato K, Zeller R, Dederichs P H, Popescu V and Ebert H 2004 Phys. Rev. B 69 054424; Mavropoulos Ph, Galanakis I, Popescu V and Dederichs P H 2004 J. Phys.: Condens. Matter 16 S5759

[35] Galanakis I, Ostanin S, Alouani M, Dreyssé H and Wills J M (2000) Phys. Rev. B 614093

[36] Galanakis I 2005 Phys. Rev. B 71012413

[37] Larson P, Mahanti S D, and Kanatzidis M G 2000 Phys. Rev. B 6212754

[38] Orgassa D, Fujiwara H, Schulthess T C, and Butler W H 1999 Phys. Rev. B 6013237

[39] Alling B, Shallcross S, and Abrikosov I A, 2006 Phys. Rev. B 73064418

[40] Picozzi S, Continenza A, and Freeman A J 2004 Phys. Rev. B 69094423 
[41] Miura Y, Nagao K, and Shirai M 2004 Phys. Rev. B 69144113

[42] Galanakis I, Özdog̃an K, Aktaş B, and Şaşıỡlu E 2006 Appl. Phys. Lett. 89 042502; Özdog̃an K, Şaşıõlu E, Aktaş B, and Galanakis I unpublished (preprint: cond-mat/0607562)

[43] Galanakis I J. Phys.: Condens. Matter 2004163089

[44] Antonov V N, Dürr H A, Kucherenko Yu, Bekenov L V, and Yaresko A N 2005 Phys. Rev. B 72054441

[45] van Leuken H and de Groot R A 1995 Phys. Rev. Lett. 741171

[46] de Groot R A, van der Kraan A M, and Buschow K H J 1986 J. Magn. Magn. Mater. 61330

[47] Şaşıŏlu E, Sandratskii L M, and Bruno P 2005 J. Phys.: Condens. Matter 17 995; Weht R and Pickett W E 1999 Phys. Rev. B 6013006

[48] Özdog̃an K, Galanakis I, Şaşığlu E, and Aktaş B 2006 J. Phys.: Condens. Matter 182905

[49] Skomski R and Dowben PA 2002 Europhys. Lett. 58, 544; Dowben P A and Skomski R 2003 J. Appl. Phys. 93 7948; idem 2004J. Appl. Phys. 957453

[50] Ležaić M, Mavropoulos Ph, Enkovaara J, Bihlmayer G, and Blügel S 2006 Phys. Rev. Lett. 97, 026404

[51] Chioncel L, Katsnelson M I, de Groot R A, and Lichtenstein A I 2003 Phys. Rev. B 68144425

[52] Itoh H, Ohsawa T, and Inoue J 2000 Phys. Rev. Lett. 842501

[53] Chioncel L, Arrigoni E, Katsnelson M I, and Lichtenstein A I 2006 Phys. Rev. Lett. 96137203

[54] Irkhin V Y and Katsnelson M I 2002 Eur. Phys. J. B 30, 481

[55] Mavropoulos Ph, Ležaić M, and Blügel S 2005 Phys. Rev. B 72, 174428 